\title{
Strength Development for Young Adolescents
}

\author{
Larry W. McDaniel \\ Department of Physical Education \& Exercise Science \\ Dakota State University \\ E-mail: larry.mcdaniel@dsu.edu \\ Allen Jackson \\ Department of Health, Physical Education \& Recreation \\ Chadron State College \\ E-mail: ajackson@csc.edu \\ Laura Gaudet \\ Department Chair \& Professor \\ Department of Psychology, Counseling, \& Social Work \\ Chadron State College \\ E-mail: lgaudet@csc.edu
}

\begin{abstract}
Participation in strength training is important for older children or young adolescences who wish to improve fitness or participate in sports. When designing strength training programs for our youth this age group is immature anatomically, physiologically, and psychologically. For the younger or inexperienced group the strength training activities may include push-ups, sit-ups, lunges, or pull-ups without barbells or dumbbells. These activities employ one's body weight as resistance. After training at this level those who demonstrate progress may move to machines that provide resistance or dumbbells. When the preparation is sports related the training goals should focus on developing power and strength related to sport specific skills. These types of exercises may be used for those who are entering puberty. Remember, muscle balance must be maintained at all times. Strength training activities should be supervised by those with education and experience in these areas. Although some injuries occur in most physical activities the American College of Sports Medicine supports young adolescences and children's participation in appropriately designed and competently supervised strength training programs. Although all participants should understand the risks and benefits of strength training, a young child should not be expected to comprehend the intricacies of muscle action. Focus on lifetime fitness and teach kids how to exercise properly. Provide a stimulating program that develops in our youth a positive attitude towards strength training and a healthy lifestyle. Benefits include increasing muscular strength, muscular endurance, body composition and sports performance. The primary objectives throughout all levels of youth training are safety, fitness, health, and enjoyment.
\end{abstract}

Keywords: Adolescents, Benefits of strength training, Designing strength programs, Muscle balance, Sports medicine, Strength training

\section{Training Young Adolescents}

Providing resistance training education to young adolescent groups is etched in our minds. For the most part it is a very enjoyable experience, but can require constant supervision and control. The older student should be allowed, with guidance, to set up a personalized program to fit specific needs. In comparison, the main goal for younger students is to develop basic training principles. Along with proper training guidelines, the main focus of any training program should center on improving levels of fitness and injury prevention.

When talking to adolescents about injuries, students often blink to prove they are still coherent, but have no earthly idea about concepts or issues. In their minds, they are 6' 6" feet tall (very tall for a young adolescent) and bullet proof. It is our responsibility to provide the guidance required to maintain participant safety and health. Although growth plate injuries are related to long bone growth, they are not as common as other injuries. Still, they may pose a threat to this age group. It is important to develop awareness about all types of injuries and risks involved in weight training. Longitudinal bone growth "depends on both proliferation and hypertrophy of chondrocytes in the growth plate with physiological limitations of bone tissue, thus a need to maintain function during this growth process (Wheeless, n.d.).

Growth plates, also known as the epiphysis, are the growing areas of the long bones of the upper and lower extremities. During human development, these areas of the body remain vulnerable to injury during adolescence with the occurrence 
of injury most prevalent among 14 to 16 year-old boys and 11 to 13 year-old girls. Older girls experience these fractures less frequently than their male counterparts due to earlier physical maturation. In young athletes, a serious joint injury is more likely to damage a growth plate than the ligaments surrounding a joint. A sprain injury in an adult can be compared to a growth plate injury in a young athlete (Panagis, Ballock, Ehrlich, Goodwin, \& Salter, 2001).

Approximately one half of all growth plate injuries occur in the lower end of the radius at the wrist. These injuries are also common to the tibia and fibula of the lower leg bones. After a growth plate injury has occurred, it is possible the injury may contribute to premature arrest of bone growth. "The affected bone grows less than it would have without the injury, and the resulting limb could be shorter than the opposite, uninjured limb. If only part of the growth plate is injured, growth may be lopsided and the limb may become crooked." (Panagis, Ballock, Ehrlich, Goodwin, \& Salter, 2001, p.2).

From 1991 to 1996, the United States Consumer Product Safety Commission, through its National Electronic Injury Surveillance System (NEISS), estimated the annual number of injuries associated with strength training as 20,940 to 26,120 for individuals under the age of 21 (Committee of Sports Medicine, 2001). Although these figures are not recent, they highlight the injury risk to those who do not receive proper resistance training or are not closely supervised. Research on growth plate injury is inconclusive, due to limited case reports involving epiphysis damage. The fact remains, these injuries are more common than we would know and are completely preventable. As professionals, we need to advise young athletes about proper technique and limited maximum lifts. Growth plate injury is typical of improperly supervised training episodes. As coaches, instructors, and trainers, we must realize that an ounce of prevention will last an athlete a lifetime (Committee of Sports Medicine, 2001).

\section{Could Strength Training Damage Bone Structure or Stunt Growth?}

A common concern for youth strength training is the belief that this exercise method can damage growth plates. Growth plates are made up of a layer of cartilage near the end of a bone where bone growth occurs. Although a few cases have been reported, there is no current evidence indicating structural damage due to strength training in adolescents. In fact, the American College of Sports Medicine states the main risk for these injuries are due to a lack of supervision and improper lifting.

The American College of Sports Medicine (ACSM), the American Academy of Pediatrics (AAP) and the National Strength and Conditioning Association (NSCA) support children's participation in appropriately designed and competently supervised strength training programs. Benefits include increasing the muscular strength of children and children's muscular endurance, body composition and sports performance improvements.

When designing children's strength training programs it is important to remember they are anatomically, physiologically, and psychologically immature. Adult strength training guidelines and training philosophies should not be used for this age group. Although all participants should understand the risks and benefits of strength training, a young child should not be expected to comprehend the intricacies of muscle action. The instructor should focus on lifetime fitness and teach children how to exercise properly. Above all, the instructor should provide a stimulating program that develops positive attitudes about strength training and healthy lifestyles in children. Generally speaking, if 7 and 8 year-old children are ready for organized sports or activities (e.g. little league baseball or gymnastics), they are ready for some type of strength training. Despite the previously held belief, that strength training is unsafe and ineffective for children, health organizations such as the American College of Sports Medicine (ACSM), the American Academy of Pediatrics (AAP) and the National Strength and Conditioning Association (NSCA) now "support children's participation in appropriately designed and competently supervised strength training programs."

\section{Basic Guidelines for Resistance Exercise Progression in Children}

Age 7 or Younger:

Introduce the child to basic exercises with little or no weight; develop the concept of a training session; teach exercise techniques; progress from body weight calisthenics, partner exercises, to lightly resisted exercises; keep the volume low.

Ages 8-10:

Gradually increase the number of exercises; practice exercise technique in all lifts; start gradual progressive loading of exercises; keep exercises simple; gradually increase training volume; carefully monitor toleration to exercise stress.

Ages 11-13:

Teach all basic exercise techniques; continue progressive loading of each exercise; emphasize exercise techniques; introduce more advanced exercises with little or no resistance.

Ages 14-15:

Progress to more advanced youth programs in resistance exercise; add sport-specific components; emphasize exercise techniques; increase volume. 


\section{Ages 16 or older:}

Move child to entry-level adult programs after all background knowledge has been mastered and a basic level of training experience has been gained.

\section{Training Guidelines for Young Adolescents}

An instructor to child ratio of at least 1 to 10 is recommended to provide adequate supervision and instruction. When children learn exercises for the first time, closer supervision by the instructor may be required. Children learn best by doing. When teaching a new exercise to a child, have the child perform the exercise under your supervision. Ensure that the training environment is free of hazards. Before classes begin, be aware of the exploratory nature of the child and remove or disassemble any broken equipment from the exercise room. The training room should be well lighted and adequately ventilated. Children are more prone to heat illness than adults; encouraged participants to drink water even if they are not thirsty.

Perform calisthenics and stretches before and after every strength training class. Begin with 1 set of 10 to 15 repetitions for 6 to 8 exercises; focus on the major muscle groups of the upper and lower body. Start with a relatively light weight and high repetitions. Increase the load and decrease the repetitions as strength improves. Beginning with relatively light loads will allow for appropriate adjustments to be made in addition to improved technique. Maximal lifting is not recommended for general conditioning purposes. Two to three training sessions per week on nonconsecutive days is sufficient. Increase the weight gradually as strength improves. Generally, a two to five pound increase in weight is consistent with a $5 \%$ to $10 \%$ increase in training intensity. Progression can be achieved by increasing the number of sets (up to 3) or number of exercises. Multi-joint exercises such as squats may be introduced into the program based on individual maturity and competencies Treat children with respect and speak with them in a language they understand. Remember that children should feel comfortable with the program and should look forward to the next workout. Strength training should be one part of a total fitness program. Keep the fun in fitness and promote lifetime health.

\section{Guidelines for Strength Training Young Athletes}

Help adolescents strive toward achieving their personal best. As coaches, parents and physicians, we need to be able to guide them so they can reach their goals without injuring themselves. One way to prevent injury is having the athlete train to increase strength and muscle flexibility through a properly developed strength training program.

What is the definition of strength training? This type of training refers to a method of conditioning designed to increase an individual's ability to exert or resist force. The goal is not to see which adolescent is the strongest, but to improve musculoskeletal strength. Strength training can mean using weights, or it can mean doing sit ups, pushups, and lunges without weights. Experts have found that strength training programs can be safe, effective and may help prevent certain sports-related injuries among young athletes. Recent findings suggest that strength training during childhood and adolescence may make bones stronger, a benefit which may last a lifetime.

For pre-adolescences in beginning weight training programs, the instructor should emphasize lifetime fitness and proper exercise techniques. Adults designing training programs should provide a stimulating environment that helps adolescents develop healthier lifestyles. When beginning conversations about physical training programs, the instructor should inform participants about the importance of a healthy, balanced diet to ensure student athletes get enough carbohydrates, protein and dietary fat to maintain energy for exercise. This is part of the healthy lifestyle image that will benefit athletes through their adult years.

When teaching adolescents' proper strength training techniques, the instructor should keep in mind they learn best by "doing". The instructor should show correct technique, and then closely supervise them to make sure they understand how to perform the movement. Pushups and sit ups are great for beginners, but as adolescents advance it is important to use weight machines or free weights to enhance their workouts to maintain interest and motivation. This progression is ideal if a trainer is available to teach correct lifting technique and supervise the athlete's progress.

Warming up and stretching should be performed before every workout. It is important to remember to warm up prior to stretching. For a beginning program, start with one set of 10-15 repetitions of 6-8 exercises that focus on the upper and lower body major muscle groups. Begin training with light weight and high repetitions. As technique and strength improve, increase the resistance and lower the number of repetitions. Two to three training sessions per week on nonconsecutive days is sufficient. Remember, strength training should be only one part of a total fitness program. Teaching young athletes the benefits of a healthy lifestyle and strength conditioning can give them the strong base on which to build their adult lives.

\section{Safety First}

Guidelines for youth strength training programs:

1) Express realistic expectations 
2) Teach positive lifestyle habits

3) Closely supervise technique

4) Give proper instruction and progression for upper and lower body exercises (i.e., bench press and leg press).

5) Give proper instruction and progression for single and multi-joint exercises (leg curl and shoulder press).

6) Allow gradual increases in volume and intensity, usually $2.5-5$ pound increments.

7) Systematically vary their strength training program for diversity and continued improvement.

8) Encourage participation in a variety of sports and activities.

\section{Contraindicated-Activities}

The catch phase is the upward movement of the bar from a resting position on the floor to chest height or the highest point in the movement of the bar. This movement involves a braking action to stop the momentum of the bar. The movement evolves as the elbows drop under the bar and the wrists move from flexion to hyperextension to absorb the force of the weight and prevent the downward movement of the bar. This process places a great deal of stress on the boney and ligamentous structures of the wrist. The catch phase of the power clean may damage bones, tendons, and ligaments that produce flexion, extension, ulnar flexion, and radial flexion of the wrist. Replace the finish of the power clean with a high pull; do not use the catch phase. An additional contraindicated power movement is the full squat. The lower phase of the full squat may damage ligaments, tendons, cartilage, and some muscle tissue that produce flexion and extension of the knee. Replace the full squat with quarter-squats or half-squats.

- $\quad$ The lowest part of the downward movement of the barbell when performing the bench press may be a contraindicated activity. Place a folded towel approximate 2-3 inches in height across the sternum. Perform the bench press by lowering the bar until it makes contact with the towel. Stop the movement and begin executing the upward ascent of the barbell. Use this technique when performing the bench press. This movement not only reduces stress on the sternum and ribs, but also decreases stress of muscles, ligaments, and tendons of the shoulder joint.

- $\quad$ Keep exercise fun; vary the routine often. Weight lifters are more likely to stick with strength training if they do not get bored by it. Results will not come overnight. Over time, you and your athlete will notice a difference in muscle strength and endurance.

\section{NSCA's Recommendations for Youth Strength Training}

- All athletes should be taught proper exercise and spotting technique. Exercises should initially be taught with no load to allow proper technique to be learned.

- All training sessions should be supervised by an experienced fitness professional.

- Each participant should be physically and emotionally prepared to participate in strength training program. Consider the athlete's mental and physical level of maturity when introducing more advanced exercises.

- All participants should have realistic expectations/goals.

- The exercise area should be safe and free from hazards.

- Every exercise session should be preceded by approximately five-10 minutes of a general warm-up; Warm up sessions should be followed by several sport specific warm-up exercises performed at light intensity.

- Equipment should be properly sized for the participant.

- Begin lifting, preferably, with body weight exercises. Athletes may also engage in basic machine exercises if they use light loads that allow athletes to complete $12-15$ repetitions.

- To encourage athletes, the program should progress from 1-3 sets of the exercises on 2-3 non-consecutive days. Each set should consist of 6-15 repetitions.

- Never increase the load being lifted by more than $5 \%$ for upper body or $10 \%$ for lower body exercises.

- Competition between participants should be discouraged as this may lead to athletes performing maximum lifts.

- At any sign of injury, strength training should be stopped, and participants evaluated prior to re-entering strength program.

- Never force participation in resistance training programs.

- Keep the program fun.

\section{A Healthy Habit for a Lifetime}

When young adolescents show interest in strength training, know these programs can be safe and effective with proper supervision. Supervision can come from a number of resources, including parents of young children. Local fitness clubs and school programs offer outstanding opportunities to acquaint young people with the benefits of resistance training, 
while instructing them in the need to follow specific principles and guidelines to minimize serious injury. Along with resistance training, stretching, and aerobic or anaerobic exercise should play a role in a well-rounded fitness program. Encourage physical activity in all children; it is a key step to becoming a healthy adult (Mayo Clinic Staff, 2006).

\section{References}

American Academy of Pediatrics. H. (2001). Strength training by children and adolescents. Retrieved August 27, 2008, from http://aappolicy.aappublications.org/cgi/reprint/pediatrics;107/6/1470.pdf.

Bergfeld, J. A. (1996-2009). Strength training for young athletes. Cleveland Clinic Sports Health, Cleveland Browns and Cleveland CAVS. Retrieved August 29, 2008, from http://www.infosports.com/clvclinic/strengthtrainingforyoung.htm.

Kreamer, W. J. \& Fleck, S. J. (1999) Strength training for young athletes. Human Kinetics. Champaign, IL.

Mayo Clinic Staff. (2008). Strength training: OK for kids when done correctly. Retrieved August 30, 2008, from http://www.mayoclinic.com/health/strength-training/HQ01010.

Paganism, J., Bullock, T., Ehrlich, M., Goodwin, R. \& Salter, R. (2001). Growth plate injuries. Health Link: Medical College of Wisconsin. Retrieved August 28, 2008, from http://healthlink.mcw.edu/article/926048658.html.

Quinn, E. (2003). Strength training for kids. National Strength and Conditioning Association's Youth Strength Summit. Savannah, GA.

Wheeless, C. (n.d.).Longitudinal growth. Wheeless' Textbook of Orthopaedics. Retrieved March 30, 2009, from http://www.wheelessonline.com/ortho/longitudinal_growth. 